\title{
Integration Type
}

National Cancer Institute

\section{Source}

National Cancer Institute. Integration Type. NCI Thesaurus. Code C41099.

Location of the integration of the engineered gene e.g. random or targeted. 